\title{
Plan de cuidados de enfermería en prematuro con enfermedad de membrana hialina
}

Juliana Gómez-Avila ${ }^{1}$

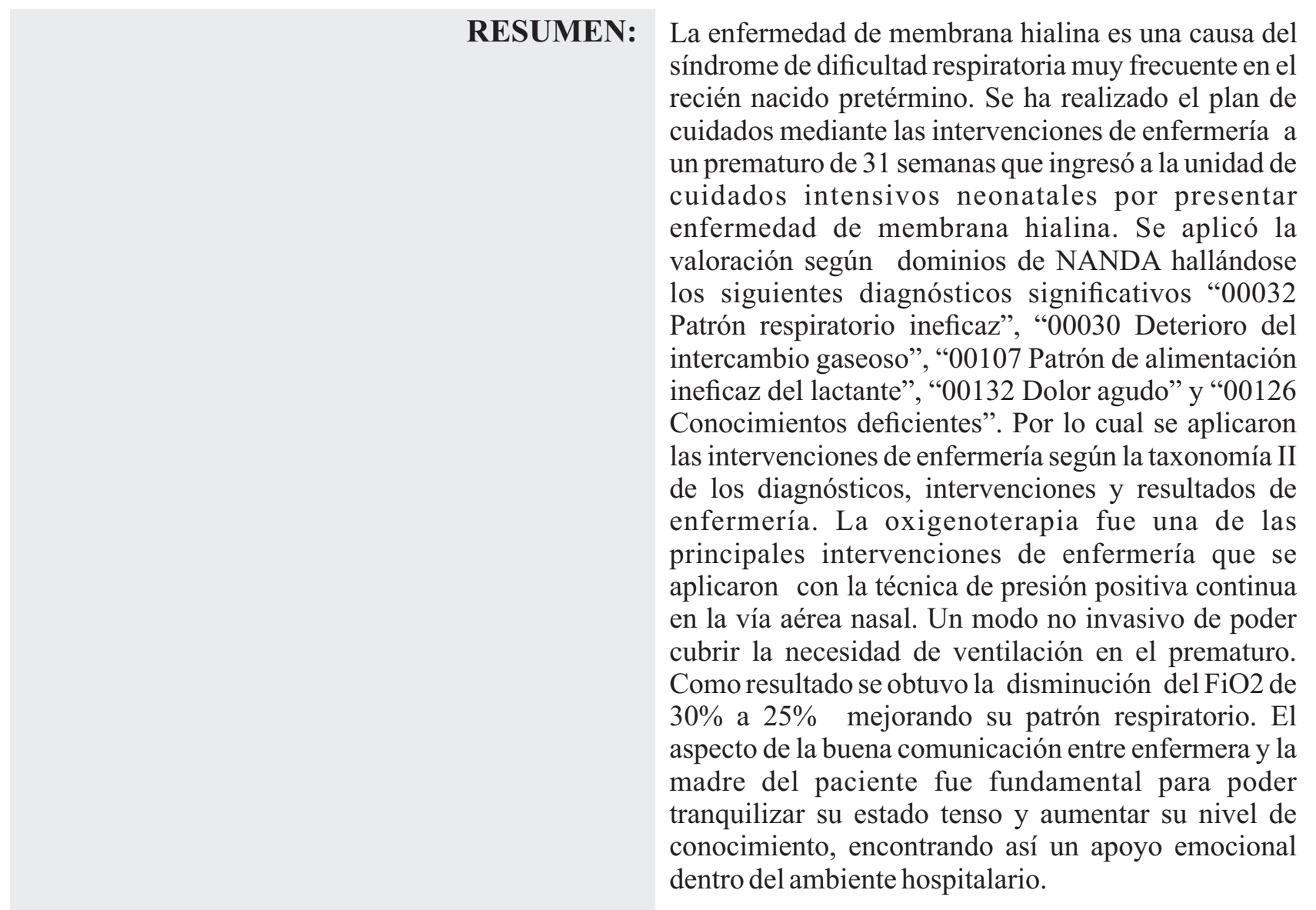

PALABRAS CLAVES: Recién nacido; Atención de enfermería; Prematuro; Oxigenoterapia.

Citar como: Gómez J. Plan de cuidados de enfermería en prematuro con enfermedad de membrana hialina. CASUS. 2016; 1(1):51-58. 


\section{INTRODUCCIÓN}

El Síndrome de dificultad respiratoria (SDR), cuya causa principal es la enfermedad de membrana hialina $(\mathrm{EMH})$, es una de las patologías respiratorias más comunes en la unidad de cuidados intensivos neonatales. Esta patología afecta fundamentalmente a los recién nacidos pretérminos (RNPT), siendo la principal causa de morbimortalidad en este grupo etario (1).

Los nacimientos pretérminos (NP) son aquellos que ocurren antes de las 37 semanas de gestación (2). En los países de ingresos bajos, una media del $12 \%$ de los niños nace antes de tiempo, frente al $9 \%$ en los países de ingresos más altos. Dentro de un mismo país, las familias más pobres corren un mayor riesgo de parto prematuro (3).

La Organización Mundial de la Salud (OMS) en el 2012 estimó que cada año nacen 15 millones de niños prematuros (uno de cada 10 nacimientos) pronosticando que esta cifra irá en aumento. En 184 países estudiados la tasa de nacimientos prematuros osciló entre el $5 \%$ y el $18 \%$ de los recién nacidos. Más del $60 \%$ de los nacimientos prematuros se producen en África y Asia meridional. Entre los países con mayor número de nacimientos prematuros se encontraron India, China, Nigeria y Brasil (3).

Según la OMS los factores que más contribuyen a la mortalidad neonatal son los RNPT. Cerca del $75 \%$ de la mortalidad perinatal y la mitad de las secuelas neurológicas a largo plazo ocurren en los pretérminos. Los mismos se complican con problemas agudos y a largo plazo: respiratorios, gastrointestinales, inmunológicos, del sistema nervioso central, de audición, visión, cognitivos y socioemocionales (2).

Aproximadamente de la mitad de los NP no se conoce la causa. Se identifican tres tipos de factores de riesgo que pueden contribuir al nacimiento pretérmino espontáneo: estrés social y raza: pobreza, baja educación, ser madre soltera, adolescente, el inadecuado control prenatal incrementan los nacimientos pretérmino y el bajo peso al nacer; infección e inflamación: la corioamnionitis que es una infección que desencadena trabajo de parto y parto pretérmino; factores genéticos: el antecedente de nacimiento pretérmino en una madre aumenta significativamente el riesgo de futuros nacimientos pretérminos (2). El prematuro con enfermedad de membrana hialina presenta diferentes complicaciones propias de la patología, sobre todo a nivel de la función respiratoria. Por lo cual, el manejo integral de enfermería en la unidad de cuidados intensivos es fundamental para poder recuperar su salud (1). Por ello la importancia de aplicar el plan de cuidados de enfermería.

La valoración del presente caso se realizó mediante los trece dominios de la clasificación de diagnósticos de North American Nursing Diagnosis Association (NANDA) (4), la Clasificación de resultados de enfermería e intervenciones de la Universidad de Iowa Nursing Outcomes Classification (NOC) (5) y Nursing Incomes Classification (NIC) (6). El objetivo del presente reporte de caso fue aplicar intervenciones de enfermería al recién nacido pretérmino con enfermedad de membrana hialina.

\section{PRESENTACIÓN DEL CASO CLÍNICO}

Recién nacido R.A.A prematuro con 31 semanas de vida de madre tercigesta de 26 años, soltera y con nivel de instrucción secundaria incompleta. La misma refirió tener condiciones económicas bajas, haber tenido solo tres controles prenatales y no contar con ningún tipo de seguro de salud. Llegó al hospital en fase expulsiva. Su hijo nace por parto eutócico pesando 1.470 gramos y con una talla de $42 \mathrm{~cm}$. Se le diagnosticó con la enfermedad de membrana hialina por lo cual fue hospitalizado en el servicio de unidad de cuidados intensivos neonatales de un Hospital Nacional del Perú.

\section{VALORACIÓN}

Se realizó la valoración mediante los 13 dominios del NANDA. Se indicó lo siguiente: el RNPT se encontraba en una incubadora con técnica de nido recibiendo oxigenoterapia en fase II - presión positiva continua en la vía aérea nasal (CPAP FiO2 al 30\%) por presentar Test de Silverman - Anderson de cinco puntos (ver Tabla 1). 
Tabla 1. Análisis de los Dominios, según aspectos positivos y negativos

\begin{tabular}{|c|c|c|}
\hline Dominios & Aspectos positivos & Aspectos negativos \\
\hline 1. Promoción de la salud & -No se observan & $\begin{array}{l}\text { Gestante de } 26 \text { años de edad llega a } \\
\text { la emergencia en periodo expulsivo, } \\
\text { con solo } 3 \text { controles prenatales, } \\
\text { RNPT AEG de } 31 \text { ss por test de } \\
\text { Capurro, recién nacido con bajo peso } \\
\text { al nacer (RNBPN): } 147 \mathrm{~g} \text {, con } \\
\text { diagnóstico médico enfermedad de } \\
\text { membrana hialina y nivel de } \\
\text { instrucción de la madre: secundaria } \\
\text { incompleta. }\end{array}$ \\
\hline 2. Nutrición & $\begin{array}{l}\text { Recibe dextrosa al } \\
10 \%+\text { gluconato de calcio } \\
\text { al } 20 \%(5.3 \mathrm{cc} / \mathrm{h})\end{array}$ & $\begin{array}{l}\text { RNPT AEG de } 31 \text { ss por Capurro } \\
\text { RNBPN: } 1470 \mathrm{~g} \\
\text { Presenta sonda orogástrica } \\
\text { Nada por vía oral (NPO) }\end{array}$ \\
\hline 3. Eliminación e Intercambio & •Micción espontánea & $\begin{array}{l}\text { Recibe oxigenoterapia } \\
\text { fase II (CPAP) } \\
\text { FiO2 al } 30 \%\end{array}$ \\
\hline 4. Actividad / Reposo & $\begin{array}{l}\text { Temperatura axilar: } 37.6^{\circ} \mathrm{C} \\
\text { Frecuencia respiratoria: } \\
58 \text { respiraciones por minuto } \\
\text { Frecuencia cardiaca: } \\
154 \text { pulsaciones por minuto } \\
\text { SatO2 } 97 \%\end{array}$ & $\begin{array}{l}\text { Se encuentra en el servicio de UCIN } \\
\text { en una incubadora con técnica de nido } \\
\text { c on S D R - E M H y r e c i b e } \\
\text { oxigenoterapia fase Il: CPAP nasal. }\end{array}$ \\
\hline 5.Percepción /Cognición & -No se observan & •No se observan \\
\hline 6. Autopercepción & -No se observan & •No se observan \\
\hline 7. Rol /Relaciones & -No se observan & $\begin{array}{l}\text { Se encuentra en UCI. } \\
\text { Falta de contacto madre e hijo. } \\
\text { Interrupción de la lactancia materna. }\end{array}$ \\
\hline 8. Sexualidad & $\begin{array}{l}\text { RNPT de sexo masculino. } \\
\text { Genitales de acuerdo a su } \\
\text { edad gestacional }\end{array}$ & •No se observan \\
\hline 9. Afrontamiento / Tolerancia al estrés & -No se observan & •No se observan \\
\hline 10. Principios vitales & -No se observan & -No se observan \\
\hline 11. Seguridad / Protección & $\begin{array}{l}\text { Se encuentra en el servicio de UCI II } \\
\text { en una incubadora con técnica de nido. }\end{array}$ & $\begin{array}{l}\text { RNPT de } 31 \text { ss recibe oxigenoterapia: } \\
\text { CPAP nasal, presenta vía periférica } \\
\text { en miembro inferior derecho. } \\
\text { n de la lactancia materna. }\end{array}$ \\
\hline 12. Confort & -No se observan & $\begin{array}{l}\text { Se encuentra en el servicio de UCI II } \\
\text { en una incubadora, prematuro } \\
\text { muestra conductas defensivas } \\
\text { cuando se le realiza algún } \\
\text { procedimiento. }\end{array}$ \\
\hline 13. Crecimiento / Desarrollo & $\begin{array}{l}\text { Recién nacido pre término AEG, } \\
\text { talla: } 42 \mathrm{~cm} \text {, perímetro cefálico: } \\
27 \mathrm{~cm} \text {, perímetro torácico: } 26 \mathrm{~cm}\end{array}$ & $\begin{array}{l}\text { RNPT, edad gestacional: Capurro } 31 \\
\text { ss. } \\
\text { RNBPN: } 1.470 \mathrm{~g}\end{array}$ \\
\hline
\end{tabular}


Debido a su mal estado de salud el RNPT fue separado de la madre, impidiéndose el contacto piel a piel y la lactancia materna precoz. En la entrevista la madre refirió no haber asistido a la totalidad de sus controles prenatales por falta de tiempo y no entender el estado de salud de su bebé.

\section{FASE DIAGNÓSTICA}

Reales:

- Patrón respiratorio ineficaz r/c con inmadurez pulmonar e/p disnea, aleteo nasal, alteración en la profundidad respiratoria $\mathrm{FiO}_{2}: 30 \%$.

- Deterioro del intercambio gaseoso $\mathrm{r} / \mathrm{c}$ cambios en la membrana alveolo capilar e/p acrocianosis, disnea, aleteo nasal $\mathrm{FiO}_{2}: 30 \%$.

- Patrón de alimentación ineficaz del lactante r/c prematuridad e/p incapacidad para coordinar la succión, la deglución y la respiración.

- Dolor agudo $\mathrm{r} / \mathrm{c}$ agentes lesivos (físico y químico) e/p conducta expresiva (llanto, inquietud), conducta defensiva y aumento de las pulsaciones.

- Conocimientos deficientes r/c desconocimiento de la enfermedad de su bebé e/p verbalización de la madre.

\section{FASE DE PLANIFICACIÓN}

Identificando los aspectos negativos se realizaron los diagnósticos de enfermería reales con NANDA. Luego se plantearon intervenciones y resultados NIC y NOC siguiendo la taxonomía II (ver Tabla 2).

\section{FASE DE EJECUCIÓN}

Se ejecutó el plan de cuidados mediante las intervenciones y actividades de enfermería según los turnos correspondientes de 12 horas.

\section{FASE DE EVALUACIÓN}

Al ejecutar las intervenciones planificadas se resolvieron los diagnósticos reales entre ellos los que comprometen la vía aérea (patrón respiratorio ineficaz y deterioro del intercambio gaseoso). Se logró mejorar el patrón respiratorio e intercambio gaseoso por medio de la oxigenoterapia con el método de CPAP nasal y la monitorización de funciones vitales. Se tuvieron como resultados: un $\mathrm{FiO} 2$ de 25\%, saturación de oxigeno $98 \%$, frecuencia respiratoria 57 por minuto, frecuencia cardiaca 140 por minuto y buena coloración de la piel. Se respondió a las interrogantes de la madre y la enseñanza se hizo efectiva mediante la correcta comunicación entre enfermera / madre para resolver el diagnóstico de conocimiento deficiente. Los demás diagnósticos y su resolución se pueden evidenciar en la Tabla 2.

\section{DISCUSIÓN}

La prematuridad trae consigo varias complicaciones en el RN. Una de las más frecuentes es el síndrome de distrés respiratorio. La enfermera debe estar capacitada para aplicar los cuidados pertinentes basándose en el proceso de cuidados de enfermería por NANDA, NIC y NOC. En este sentido es fundamental aportar evidencia de la atención de enfermería con el empleo de esta metodología. El aporte empírico de un uso efectivo de la misma es significativo para la labor del personal de enfermería y para optimizar la condición de salud del paciente.

Dentro del plan de cuidados de enfermería se encuentra el tratamiento de la oxigenoterapia con el método de CPAP nasal. Este fue empleado para mejorar el patrón respiratorio del prematuro ya que en un primer momento presentaba un $\mathrm{FiO}_{2}$ de $30 \%$ y después de aplicar las intervenciones presentó un $\mathrm{FiO}_{2}$ de $25 \%$. Según estudios se evidencia que este procedimiento es muy efectivo para evitar la ventilación mecánica, el uso de surfactante y posibles complicaciones como displasia broncopulmonar(7).

Los cuidados nutricionales forman también parte importante de la atención al prematuro. El inicio de la infusión de glucosa debe estar dentro de la hora del nacimiento, ya que los depósitos de glucógeno son escasos, se agotan rápidamente y son su principal fuente de energía celular. Por tanto es fundamental la intervención para mejorar el patrón de alimentación $(1,9)$. Frente a dicho cuidado se debe considerar el buen mantenimiento del catéter periférico, la correcta infusión, el balance hidroelectrolítico e inicio precoz de la técnica de extracción de leche materna, para poder disponer de ella a penas se indique la alimentación enteral mínima $(8,9)$.

Respecto al manejo del dolor en el RNPT la literatura indica que una correcta valoración se basa en la observación de la conducta del mismo. A su adecuada valoración puede seguir tanto un tratamiento farmacológico como el uso de técnicas no farmacológicas (10). Entre estas últimas destacan la optimización del macroambiente y el control del 


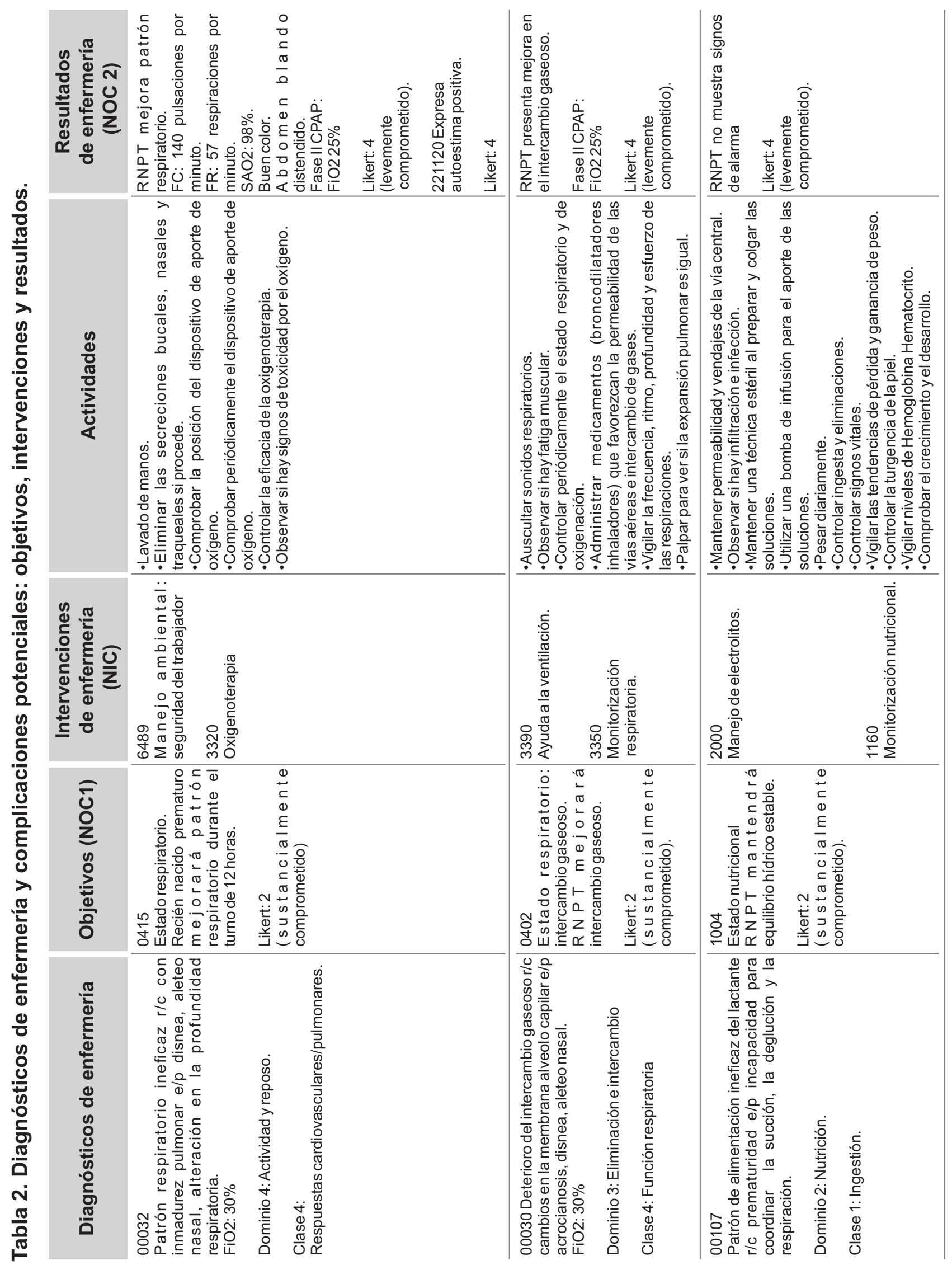




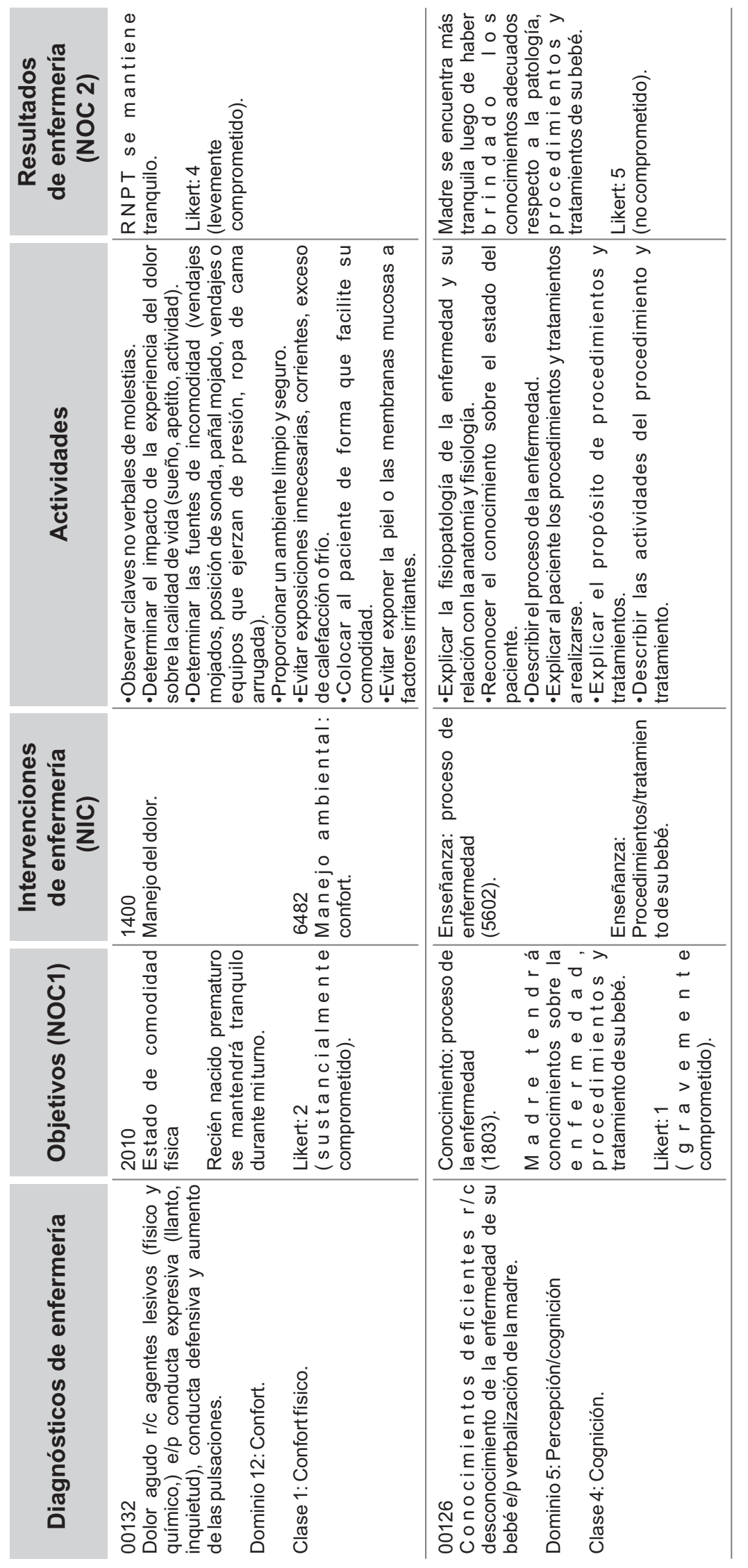


microambiente (manipulación y posicionamiento) (10). Por otro lado, un manejo adecuado del dolor trae consigo que el prematuro tenga un menor gasto de energía, signos vitales más estables, aumento de peso entre otros beneficios. Este estado de organización impacta directamente en la evolución de la enfermedad, ya que un RN con el diagnóstico mencionado aumenta el consumo de oxígeno y empeorara su condición respiratoria (1).

Otro aspecto a considerar es el estado emocional de los padres, en particular de la madre vinculado a los escasos conocimientos relativos a las necesidades de su hijo prematuro (11). El desconocimiento y la dificultad de interactuar físicamente con el mismo generan pérdida de autoestima y sensación de poco control (11). Por tales motivos el mantener un lenguaje sencillo y entendible permitirá a la enfermera continuar el vínculo, identificar dudas y preocupaciones para poder responder ante ellas. Estas intervenciones educativas deben incluir a los padres en el cuidado del prematuro y conciben a la familia como unidad de cuidados (12).

El RNPT con enfermedad de membrana hialina significa un desafío para el cuidado integral de enfermería neonatal en la unidad de cuidados intensivos neonatales. La aplicación del plan de cuidados de enfermería utilizando NANDA, NIC y NOC permitirá obtener resultados positivos, por ello se recomienda hacer uso de esta metodología en el campo de la enfermería neonatal.

\section{REFERENCIAS BIBLIOGRÁFICAS}

1. Quiroga A. Cuidados al recién nacido con síndrome de dificultad respiratoria. Plan de cuidados de enfermería. Enfermería Neonatal. 2013;(16):4-9.

2. Organización Panamericana de la $\mathrm{S}$ a lud. A I E P I N e on at a 1: Intervenciones Basadas en Evidencia. 2da ed. Washington, D.C: OPS; 2009.

3. March of Dimes, PMNCH, Save the Children, Organización Mundial de la Salud. Nacidos Demasiado Pronto: Informe de Acción Global sobre Nacimientos Prematuros. Nueva York: Howson CP, Kinney MV, Lawn JE; 2012. 1-8.

4. Herdman TH, Kamitsuru S. NANDA International. Diagnósticos Enfermeros: Definiciones y Clasificación, 2015-2017. Barcelona: ElSevier; 2015.

5. Moorhead S, Johnson M, Maas ML, Swanson E. Clasificación de Resultados de Enfermería (NOC). 5ta ed. Barcelona: Elsevier; 2014.
6. Bulechek GM, Butcher HK, Dochterman JM, Wagner CM. Clasificación de Intervenciones de Enfermería (NIC). 6ta ed. Barcelona: Elsevier; 2014.

7. Osorno L. Papel actual de la presión positiva continua en la vía aérea en el síndrome de dificultad respiratoria y nuevas evidencias. Bol Med Hosp Infant Mex. 2012;69(6):422-430.

8. González C, Omaña MF. Síndrome de distrés respiratorio neonatal o enfermedad de membrana hialina. Bol Pediatr. 2006; 46(1): 160-165.

9. Mena P, Milad M, Vernal P, Escalante MJ. Nutrición intrahospitalaria del prematuro. Recomendaciones de la Rama de Neonatología de la Sociedad Chilena de Pediatría. Revista Chilena de Pediatría. 2016;87(4):305-321.

10. González C, Fernández I. Revisión bibliográfica en el manejo del dolor neonatal. ENE. Revista de Enfermería. Dic. 2012;6(3).
11. Cockcroft S. How can family centred care be improved to meet the needs of parents with a premature baby in neonatal intensive care? Journal of Neonatal Nursing. 2012;18(3): 105110.

12. Petruskavich S, Manikandan R, Schallers D. Leading the Baby Friendly Initiative in a NICU: One Hospital's Journey to Ignite the Change. Newborn and Infant Nursing Reviews. 2013;13(3):133-136. 


\section{Nursing care plan in premature with hyaline membrane disease}

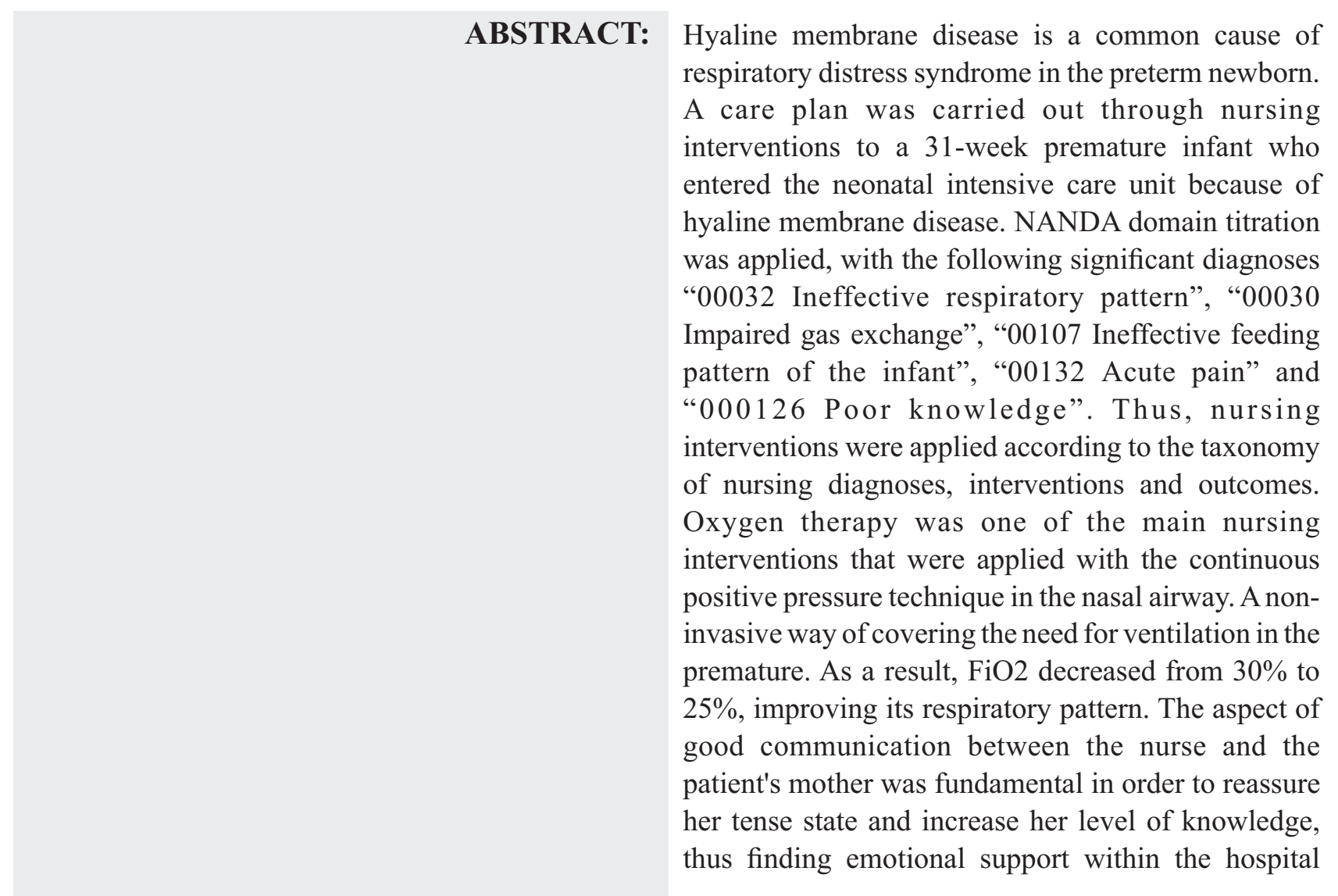

KEY WORDS: Newborn; Nursing care; Premature; Oxygen therapy.8 